\title{
LES NANOSTRUCTURES POUR CRÉER DE LA COULEUR un art inspiré par la nature
}

\author{
Extrait de Photoniques, \\ hors-série Année internationale \\ de la Lumière (2015) \\ D'après les travaux de \\ Philip BALL \\ auteur de Nature's color tricks \\ (Sci. Am. 306, 74-79, 2012) \\ p.ball@btinternet.com
}

Les reflets changeants des plumes de la queue du paon ont captivé plus d'un esprit curieux. Le scientifique anglais Robert Hooke les qualifiait en 1665 de "surnaturelles » en constatant que, mouillées, elles perdaient leurs couleurs. À l'aide du microscope inventé depuis peu, il observa ces plumes, et découvrit qu'elles étaient couvertes de stries - qu'il suspecta d'être à l'origine des couleurs. Aujourd'hui, la recherche tente de s'inspirer de ces phénomènes : des applications «bio-inspirées » sont en voie de concrétisation. et nanostructures dédiées. Ces mécanismes biologiques inspirent les ingénieurs utilisant la lumière dans des technologies optiques : ils pourraient servir à la mise au point de dispositifs d'affichage plus lumineux, de nouveaux capteurs chimiques, ou encore de solutions de transport et traitement de l'information. Pour faire un usage optimal de ces phénomènes, il nous faut en premier lieu comprendre comment la nature crée et met en œuvre ces minuscules structures optiques.

Si le mystère persiste autour de la diversité de mécanismes existant dans la nature, et de la façon dont ils ont évolué, il est clair néanmoins que la nature ne dispose pas des technologies de fabrication sophistiquées, telle la gravure par faisceaux d'électrons employée (Vukusic \& Sambles, 2003 ; Vukusic, 2004 ; Wolpert, 2009). Cette dispersion se produit pour des longueurs d'ondes spécifiques, qui sont fonction de la taille et de l'espacement des structures, et correspondant à des couleurs précises du spectre. Les différentes nuances peuvent ensuite dépendre de l'angle d'observation : les couleurs structurelles sont souvent iridescentes, fluctuant du bleu au vert, ou du jaune à l'orange. Impliquant un phénomène de réflexion et non d'absorption, elles se singularisent par leur éclat. Ainsi les papillons Morpho bleus d'Amérique du sud et d'Amérique centrale sont visibles à plusieurs centaines de mètres, et semblent presque briller dès que le les rayons du soleil traversent la canopée pour se réfléchir sur leurs ailes.

Les couleurs structurelles illustrent la capacité des organismes vivants à manipuler la lumière à l'aide de micro

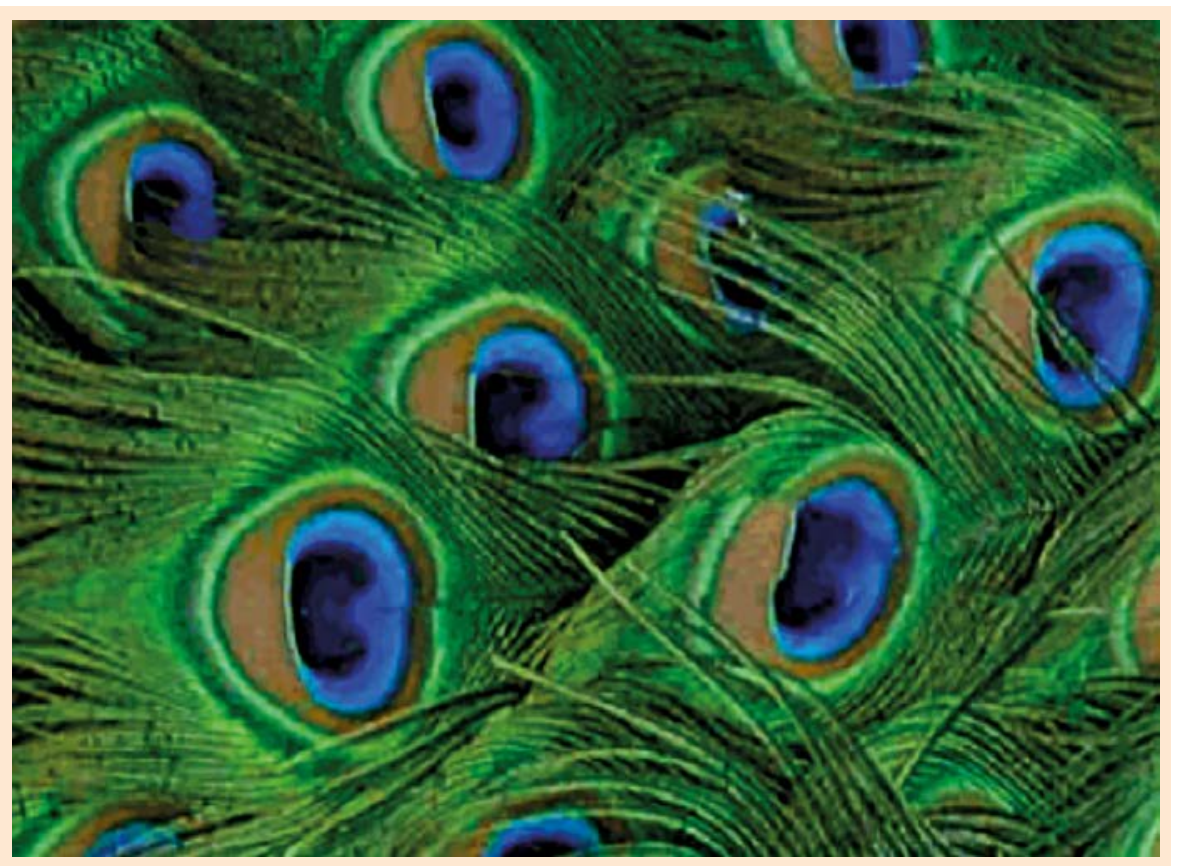

Figure 1. Les couleurs changeantes de la queue du paon ont fait l'objet, depuis des siècles, de nombre d'interprétations métaphoriques. 
pour sculpter des structures optiques à partir de blocs de matière. Les organismes vivants, fabriquent leurs structures à partir de briques élémentaires.

En maîtrisant ce domaine, nous pourrions développer des technologies bon marché pour fabriquer par exemple, des matériaux qui changent de couleur ou d'apparence à la manière de certains poissons ou calmars, des fibres qui transportent la lumière sans pertes, ou encore des obturateurs contrôlés chimiquement. Voici une présentation de stratagèmes utilisés par la nature pour créer la couleur à partir de structures nanométriques ; et de dispositifs et matériaux artificiels qui s'en sont inspirés.

\section{Des empilements de nanostructures organisées}

Bien que les sillons observés par Hooke dispersent bien la lumière, les teintes vives prises par la lumière réfléchie proviennent généralement de structures invisibles situées sous la surface. Dans la nature, cette solution constitue une méthode fiable pour générer des couleurs, qui ne dépend pas de pigments organiques, fragiles et photosensibles.

Les écailles et plumes des oiseaux, poissons et papillons comportent habituellement des surfaces ou des aiguilles microscopiques, faites d'un matériau dense offrant des propriétés de dispersion de la lumière, lui-même contenu dans une matrice d'un autre matériau. La distance entre les structures étant proche des longueurs d'onde de la lumière visible, leur empilement crée un phénomène ondulatoire de diffraction, dans lequel les ondes réfléchies interferent les unes avec les autres. Suivant l'angle de réflexion, les rayons lumineux d'une certaine longueur d'onde interferent au contact des couches successives de l'empilement, amplifiant la couleur correspondante dans la lumière réfléchie (Vukusic \& Sambles, 2003; Vukusic, 2004; Wolpert, 2009). Le phénomène est similaire à celui qui fait apparaître le spectre chromatique sur un CD lorsqu'on l'incline.
Sur les ailes des papillons, les empilements réflectifs séparés par des couches d'air sont constitués d'un polymère naturel, la chitine. Dans les plumes des oiseaux, ces empilements pigmentés par de la mélanine, ont la forme d'aiguilles ou de plaquettes ; parfois de cavités recouvertes de kératine, comme chez le colibri Coeligena prunellei. En optique, des réseaux de diffraction analogues, formés d'une alternance de deux matériaux en couches ultra-minces sont $\mathrm{em}$ ployés dans les dispositifs permettant de sélectionner et réfléchir les rayons lumineux d'une couleur donnée. Par exemple, les miroirs de Bragg constitués de couches de semi-conducteurs jouent le rôle de réflecteurs et filtres de couleurs, dans des appareils allant des télescopes astronomiques aux spectromètres et lasers solides.

Le bleu spectaculaire des papillons Morpho didius et Morpho rhetenor provient non pas d'un simple empilement de couches, mais de nanostructures plus complexes contenues dans leurs écailles : un arrangement de structures composées de chitine, dressées en surface comme des arbres de Noël (Vukusic \& Sambles, 2003). Chaque 'arbre', formé d'une pile de strates circulaires, agit comme un réseau de diffraction. Ses surfaces peuvent réfléchir jusqu'à $80 \%$ de la lumière bleue incidente. Et, comme elles ne sont pas plates, elles peuvent renvoyer la même couleur suivant différents angles de vue, limitant ainsi le phénomène d'iridescence; en effet, les animaux ne souhaitent pas toujours que leur couleur change en fonction de la direction depuis laquelle ils sont vus.

La couleur réfléchie dépend en fait de la différence d'indice de réfraction entre les nanostructures et le milieu environnant. Celui-ci est habituellement l'air, mais, comme l'a observé Robert Hooke, mouiller de telles surfaces impacte la différence d'indices, et modifie la couleur de diverses façons, suivant l'indice de réfraction du liquide mouillant. Exploitant cette propriété, des structures de synthèse obtenues par technique de gravure microlithographique sont élaborées par des chercheurs de GE Global Research
(New York) en collaboration notamment avec le spécialiste des ailes de papillons Pete Vukusic (université d'Eaxter, Royaume-Uni). Utilisées comme capteurs chimiques de changement de couleur, elles doivent permettre d'identifier différents liquides; des applications sont possibles en détection des émissions polluantes de centrales de production d'électricité, en sécurité alimentaire et en analyse de la qualité de l'eau.

\section{Des cristaux photoniques 《nanoéponges »}

La dispersion par des arrangements ordonnés d'objets microscopiques peut, pour certaines configurations, faire disparaittre la lumière dans une plage de longueurs d'ondes donnée, appelée bande interdite (Vukusic, 2004). De tels objets baptisés « cristaux photoniques » se rencontrent à l'état naturel, dans l'opale par exemple - une forme de silice dans laquelle la dispersion est opérée par de minuscules sphères minérales. Des cristaux photoniques artificiels ont été mis au point pour canaliser la lumière, dans des guides d'ondes associés à des puces silicone, employés dans les télécom optiques.

La technique est déjà maîtrisée par des organismes vivants. Observées au microscope électronique, les écailles des ailes du papillon Parides sesostris laissent apparaître des arrangements formant des chevrons : ces carreaux de chitine, dont la surface présente un aspect évoquant une éponge, sont perforés de façon régulière, avec des cavités d'un diamètre de l'ordre de 100 nanomètres. Chaque carreau est un cristal photonique. Michielsen et Stavenga ont remarqué que ces cristaux, présents sur les ailes de P. sesostris et d'autres papillons, ont une structure décrite comme «gyroïde » par les mathématiciens (Michielsen \& Stavenga, 2008). Les structures observables à la surface des ailes de P. sesostris ont une bande interdite qui leur permet de réfléchir la lumière dans la partie verte du spectre, sur une large plage d'angles d'incidence (figure 2). Certains charançons, 

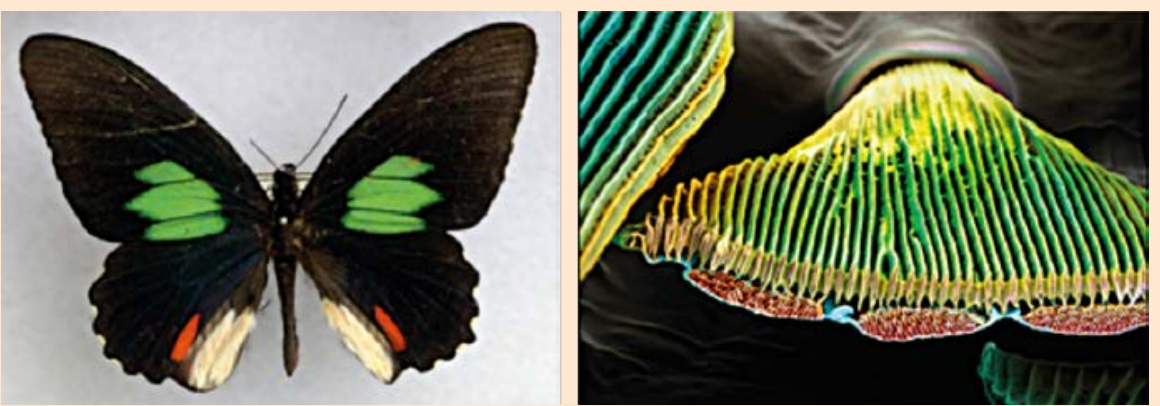

Figure 2. Les écailles des ailes de P. sesostris contiennent des cristaux photoniques de chitine.

comme d'autres scarabées, tirent leur couleur irisée de ce type de cristaux photoniques tri-dimensionnels.

Pour synthétiser des structures similaires, il est possible de recourir à des composés surfactants - dotés de propriétés tensio-actives similaires à celles des lipides - ou à des copolymères. Ulrich Wiesner et ses collaborateurs (Stefik et al., 2012) ont associé des copolymères liquides avec des nanoparticules de niobium et d'oxyde de titane ; les polymères ont formé des gyroïdes ainsi que d'autres structures de type 'nano-éponge', arrangeant les nano-particules suivant les mêmes schémas. Le matériau polymère ayant servi de matrice est ensuite éliminé par combustion, avec pour résultat un arrangement continu de nanoparticules.

Ces objets poreux peuvent se prêter à de nombreuses utilisations. Des films minces poreux de dioxyde de titane sont employés dans le domaine du photovoltaiqque, comme revêtement peu coûteux pour les cellules solaires. Leurs réseaux giroïdes ordonnés présentent l'avantage, par rapport à un contact électrique entre nanoparticules aléatoires, de constituer un matériau conducteur continu pour le transport des électrons. Des calculs ont montré (Hur et al., 2011), que des nano-éponges à structure double-giroïde constituées de métaux tels que l'argent ou l'aluminium, fabriquées à partir de nano- particules organisées dans une matrice de copolymères, peuvent présenter un indice de réfraction négatif. Ces matériaux pourraient faire office de «superlentilles » pour microscopes optiques, qui permettraient alors d'imager des objets de taille inférieure à la longueur d'onde de la lumière. S'inspirant des structures mises en œuvre par les papillons, Turner et al. (2011) ont utilisé un laser pour graver ces cristaux photoniques dans une résine photosensible. Présentant des bandes interdites dans l'infrarouge, ce matériau trouve des applications dans les télécoms; la variante dotée d'un maillage en tire-bouchons répond différemment à une lumière polarisée circulairement gauche, ou droite.

\section{Des nanostructures désordonnées pour créer de nouveaux matériaux opaques}

La plupart des oiseaux ont un plumage bleu ou vert dû à des nanostructures optiques, non iridescent à l'inverse de celui du paon ou du colibri : ils présentent la même couleur, quel que soit l'angle sous lequel on les observe. Leurs nanostructures de type nano-éponge, faites de kératine, sont désordonnées : elles ne réfléchissent pas la lumière, mais la dispersent de façon diffuse (Dufresne et al., 2009).

Les nanostructures mises en jeu apparaitraient lors de la séparation de la kératine et du cytoplasme cellulaire à l'origine de la formation des plumes, similaire à une séparation de phases eau/huile. Dans des mélanges tels que les alliages de métal ou les polymères en cours de solidification, une séparation de phases crée, en fonction des conditions, des arrangements différents. Dans le cas d'un mélange instable, les composés se séparent pour donner des structures désordonnées et interconnectées, suivant un phénomène appelé décomposition spinodale. En revanche si le mélange est métastable, comme peut l'être de l'eau sursaturée
Wavefront sensors and adaptive optics for optical metrology, lasers and microscopy
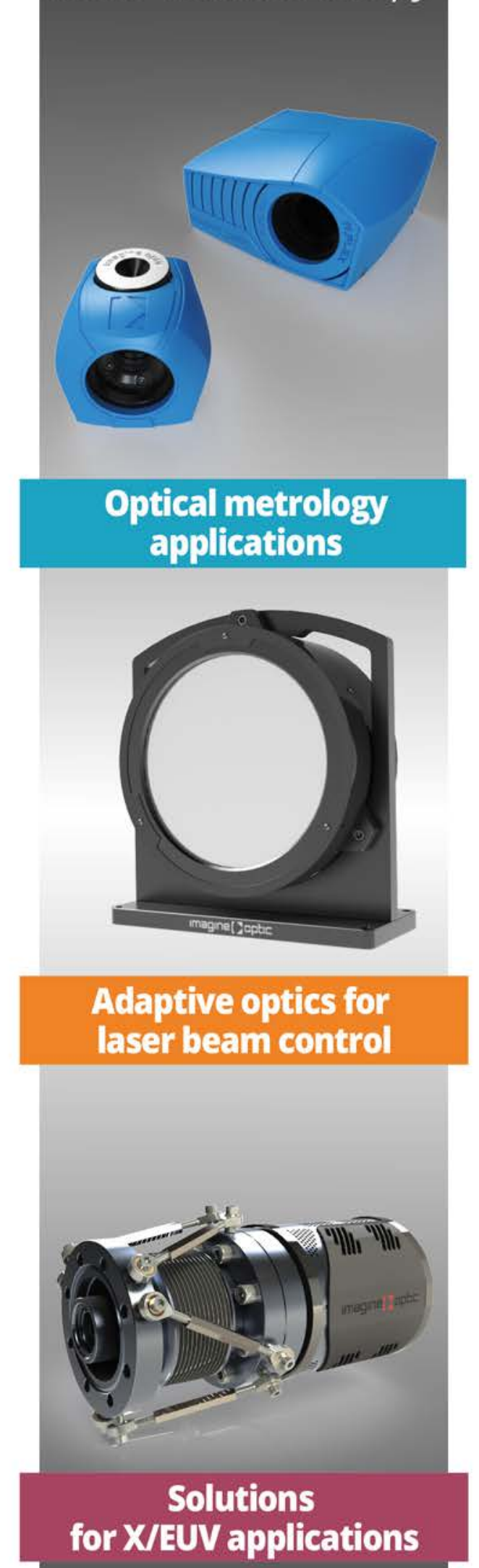

Contact us for more details: contact@imagine-optic.com or +33164861560 


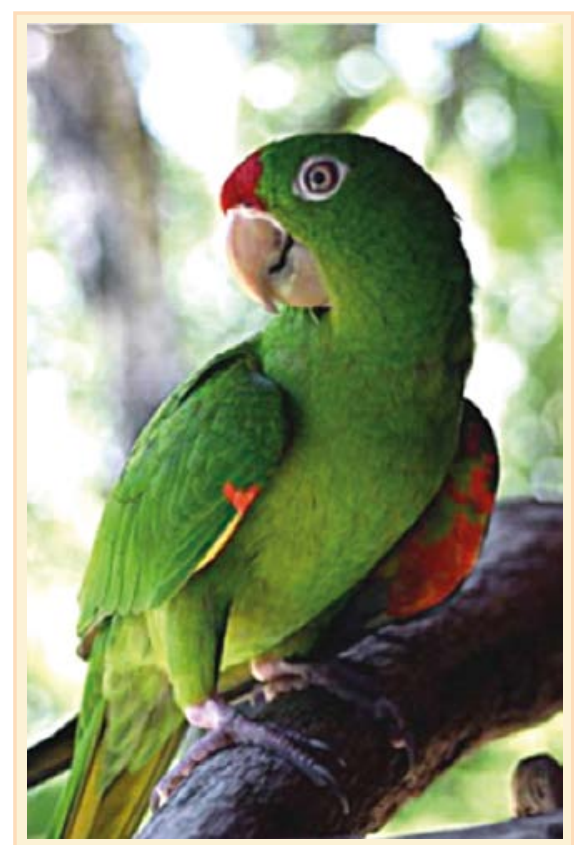

Figure 3. La couleur verte caractéristique des perroquets est due à la combinaison de la couche réflective bleue de leurs plumes, avec un pigment jaune.

en gaz dissous, alors la séparation de phase va donner naissance à des structures disjointes. L'un ou l'autre de ces processus serait à l'œuvre dans la formation des plumes des oiseaux, auxquels l'évolution aurait conféré la faculté de contrôler la croissance des nanostructures ainsi fabriquées, pour la stopper au stade correspondant à une longueur d'onde de lumière dispersée - donnant au plumage la couleur souhaitée. Un mécanisme similaire peut être observé dans la couleur et l'opacité du lait (gouttelettes de matière grasse de tailles diverses en suspension). Les verriers ont pendant des siècles, utilisé la précipitation de nanoparticles d'or dans la silice, pour fabriquer des vitraux de couleur rubis.

Aujourd'hui, les ingénieurs s'intéressent à la façon d'utiliser ces structures pour créer des matériaux colorés fortement opaques. Vukusic et ses collègues se sont inspirés de la carapace du coléoptère Cyphochilus, d'un blanc brillant particulier, dû à un réseau désordonné de canaux de type 'nano-éponge' (Hallam et al., 2009). Ils ont fabriqué des réseaux poreux aléatoires de filaments interconnectés de carbonate de calcium et de dioxyde de titane, mélangés à une huile et à un polymère jouant le rôle de liants, éliminés après séchage : en s'approchant de la taille et de la densité des filaments observés chez le coléoptère, il leur a été possible de parvenir à des revêtements fins, d'une blancheur et d'une brillance similaires. Des chercheurs de l'université de Yale (Forster et al., 2010) ont quant à eux imité les nanoéponges désordonnées des plumes d'oiseaux, pour créer des films formés de microscopiques chaînes de polymères disposées aléatoirement, présentant une couleur bleu-vert.

\section{Une combinaison de mécanismes encore mystérieuse}

La plupart des effets rencontrés dans la nature ne sont pas dus uniquement à des couleurs « structurelles ", mais à leur combinaison avec des pigments (Shawkey et al., 2009). Une fine couche de pigments au-dessus de la couche réflective du calmar peut par exemple, faire apparaittre des taches. Dans les plumes des oiseaux, les couleurs résultant des nanostructures de mélanine contenues dans leur matrice de kératine, peuvent être modulées par des pigments tels les caroténoïdes, qui absorbent la lumière rouge et la lumière jaune (figure 3).

\section{POUR EN SAVOIR PLUS}

[1] Ball P., "Nature's color tricks", Sci. Am. 306, 74-79 (2012).

[2] Dufresne E. R., Noh H., Saranathan V., Mochrie S. G. J., Cao H., Prum R. O., Soft Matter 5, 1792-1795 (2009).

[3] Forbes P. (2009), Dazzled and Deceived: Mimicry and Camoulage. Yale University Press, New Haven.

[4] Forster J. D., Noh H., Liew S. F., Saranathan V., Schrenk C. F., Yang L., Park J-G, Prum R. O., Mochrie S. G. J., O'Hern C. S., Cao H., Dufresne E. R., Adv. Mater. 22, 2939-2944 (2010).

[5] Hallam B. T., Hiorns A. G., Vukusic P., Appl. Opt. 48, 3243-3249 (2009).

[6] Hur K., Francescato Y., Giannini V., Maier S. A., Hennig R. G., Wiesner U., Angew. Chem. Int. Ed. 50, 11985-11989 (2011).

[7] Michielsen K., Stavenga D. G., J. R. Soc. Interface 5, 85-94 (2008).

[8] Schenk F., Parker A., Leonardo 44, 108-115 (2011).

[9] Shawkey M. D., Morehouse N. I., Vukusic P., J. R. Soc. Interface 6, S221-S231 (2009).

[10] Steik M., Wang S., Hovden R., Sai H., Tate M. W., Muller D. A., Steiner U., Gruner S. M., Wiesner U., J. Mater. Chem. 22, 1078-1087 (2012).

[11] Turner M. D., Schröder-Turk G. E., Gu M., Opt. Express 19, 10001-10008 (2011).

[12] Vukusic P., Sambles J. R., Nature 424, 852-855 (2003).

[13] Wolpert H. D., Optics and Photonics News 20, 22-27 (2009). 